\title{
Heavy Quark Potential at Finite Temperature in a Dual Gravity Closer to Large N QCD
}

\author{
Binoy Krishna Patra and Himanshu Khanchandani \\ Department of Physics, Indian Institute of Technology Roorkee, Roorkee 247667 , India
}

\begin{abstract}
In gauge-gravity duality, the heavy quark potential at finite temperature is usually calculated with the pure AdS background, which does not capture the renormalization group (RG) running in the gauge theory part. In addition, the potential does not contain any confining term in the deconfined phase. Following the Klebanov-Strassler geometry, we employ a geometry, which captures the RG flow similar to QCD, to obtain the heavy quark potential by analytically continuing the string configurations into the complex plane. In addition to the attractive terms, the obtained potential has confining terms both at $T=0$ and $T \neq 0$, compared to the calculations usually done in the literature, where only the Coulomb-like term is present in the deconfined phase. The potential also develops an (negative) imaginary part above a critical separation, $r_{c}\left(=0.53 z_{h}\right)$. Moreover, our potential exhibits a behavior different from the usual Debye screening obtained from perturbation theory.
\end{abstract}

PACS: $\quad$ 12.39.-x,11.10.St,12.38.Mh,12.39.Pn

Keywords: Heavy quark potential, Wilson loop, Thermal width, AdS/CFT, Nambu-Goto Action

\section{Introduction}

The heavy quarks produced in the early stage of relativistic heavy-ion collisions (HIC) is one of the crucial probes to the medium formed at later stage of the collision, known as quark-gluon plasma (QGP). Matsui and Satz [1] first proposed the idea of Debye screening of the potential between a heavy quark and a heavy antiquark, which causes the suppression of the yields of heavy quarkonium states in HIC [2]. Since then many efforts have been devoted to understand the change of properties of $Q \bar{Q}$ states in the deconfined medium, using either the non-relativistic 
calculations based upon some effective (screened) potential $[9,10,18,11,12,13]$ or the lattice calculations of quarkonium spectral functions $[3,4,5,6,7,8]$. Potential models provide a simple and intuitive framework for the study of quarkonium properties at finite temperature but the main problem is the determination of the effective potential. At zero temperature, the use of effective potentials and their connection to the underlying field theory is well established but at finite temperature the issue is still open $[14,15,16]$. Different potential models based upon the lattice free and internal energies have a fair agreement with the results from the lattice studies $[18,12]$ whereas the calculations of Euclidean correlators using a variety of potential models were not able to reproduce the temperature dependence of the lattice correlators $[17,11,12]$. It was not until recently that effective field theory (EFT) approaches have been developed to justify the use of potential models at finite temperature because the EFT techniques are suited for handling systems with well separated energy scales. Two types of scales appear viz. at $\mathrm{T}=0$ : the mass of the heavy quark, $m_{Q}$, the typical momentum transfer $\left(m_{Q} v_{Q}\right.$, and the binding energy $\left(m_{Q} v_{Q}^{2}\right)$, and at $T \neq 0$ : the temperature $T$, the Debye mass $m_{D}(=g T)$, and $g^{2} T[19,20]$. Since the relative velocity $\left(v_{Q}\right)$ is very small $\left(v_{Q} \sim \alpha_{s}<1\right)[21,22]$, both types of scales are well separated.

Nowadays it is understood that the dissociation of heavy quarkonia is not due to the Debye screening of the potential, but is rather overtaken by the thermal width obtained from the imaginary part of the potential $[23,19,24]$. There are two different processes in QCD, which contribute to the thermal width: the first process is the inelastic parton scattering mediated by the space-like gluons, when the Debye mass is greater than the binding energy. This process is often referred as Landau damping and is the principal source of dissociation mechanism at weak coupling. The second process is the gluon-dissociation process which corresponds to the decay of a color singlet state into a color octet induced by a thermal gluon and is dominant when the binding energy is greater than the Debye mass [25]. Recently one of us derived both the real and the imaginary component of the potential by correcting both the perturbative and nonperturbative (string) terms of the potential at $\mathrm{T}=0$ in real-time formalism. We found that after inclusion of confining string term, the (magnitude) imaginary component becomes larger and hence contribute more to the thermal width, compared to the medium-modification of the perturbative term alone $[26,27,28]$ However, the above discussions are limited to the weak coupling techniques.

The large and anisotropic collective flow and its strong interaction with the hard probes at 
collider experiments indicate that the matter created must be described by QCD in a regime of strong interactions. In the strong coupling regime, the effect of an imaginary part in usual potential models has been addressed in [29], and also in the T-matrix approach through the Schwinger-Dyson equations [30]. The imaginary part has also been calculated on the lattice $[49,31]$, where the spectral functions have been extracted, using the limited sets of data. This fact, together with the intrinsic technical difficulties of lattice calculations, somehow limits the reliability of the results obtained so far, and also their scope. Therefore some complementary methods for strong coupling at finite temperature are desirable. The AdS/CFT conjecture provides such an alternative $[32,33,34]$ for a class of non-abelian thermal gauge field theories, which gives an analytic access to the strong coupling regime of finite temperature gauge field theories in the limit of large number of colors. This is achieved by mapping the problems at strong coupling onto calculable problems in the supergravity limit of a dual string theory. The background metric will be described by a curved five-dimensional AdS spacetime containing a black hole with its horizon lying in the fifth dimension. Although the AdS/CFT correspondence is not directly applicable to QCD, but the universality of the result for the shear viscosity-to-entropy ratio with the prediction of hydrodynamics in HIC [35] motivates to calculate the heavy quark potential at finite temperature.

The expectation values of Wilson loops contain gauge invariant information about the nonperturbative physics of non-abelian gauge field theories. In fact, the expectation value of a particular time-like Wilson loop serves to define the potential between a static quark and antiquark at finite temperature. The first calculation was done by Maldacena [36] for $\mathcal{N}=4 \mathrm{SYM}$ for $\mathrm{T}=0$ and was later extended to finite temperature in $[37,38]$. The potential is obtained by extremizing the world sheet of open string attached to the heavy quark and its anti quark located at the boundary of $\mathrm{AdS}_{5}$ space in the background of $\mathrm{AdS}_{5}$ space [36] and in the background of $\mathrm{AdS}_{5}$ black-hole metric $[37,38]$, for determining the potentials at $T=0$ and $T \neq 0$, respectively. The problem with pure AdS background is that it does not capture the renormalisation group running, like in QCD and does not give any confining term in the potential. In this work we use the Ouyang-Klebanov-Strassler-Black-Hole (OKS-BH) geometry [39] explored recently in $[40,43,44]$, which captures the logarithmic running of the coupling constants at far IR and is almost conformal at UV and calculate both real and imaginary parts of the potential at finite temperature by analytically continuing the string configurations into the complex plane. Our 
work is organised as follows. In Section 2, we have revisited the OKS-BH geometry in brief and in Section 3, we employ this geometry to construct the Nambu-Goto action for obtaining the potential. Finally we conclude in Section 4.

\section{Geometry}

Let us suppose a gauge theory living in $3+1$ dimensions defined at a particular energy scale $\Lambda$. It can be thought of as being defined on a $3+1$ dimensional slice in a higher dimensional space and if we stack up all such slices together we can have a continuous family of gauge theories labelled by $\Lambda$. We can imagine this continuous family as a single theory in five dimensions with $\Lambda$ becoming the fifth dimension. The flow along this energy scale is known as renormalisation group (RG) flow. Now if the theory is conformal i.e. does not flow with energy $(\Lambda)$, it can be described on the boundary of a five-dimensional space. In other words a conformal field theory (CFT) can be described conveniently on the boundary of pure Anti-de Sitter (AdS) space [32]. But if the theory has a RG flow we cannot describe the full theory simply by describing it on the boundary of some five dimensional space and we need to look differently at different energy scales. In particular for large N QCD, we have conformal behaviour at UV and logarithmic running of coupling at IR and we need to look differently at these two regimes. The geometries that can account for such a RG flow have been constructed and discussed in detail in [43, 40]. We shall briefly review them here.

It has been seen that if we embed D branes in a geometry, the gauge theory living on these D branes may show logarithmic RG flow. A particular model that does this is the KlebanovStrassler (KS) warped conifold construction [39]. The gravity dual of this construction, which is a warped deformed conifold with three form type IIB fluxes, captures the RG flow of the gauge theory. The corresponding gauge theory is confining in the far IR but is not asymptotically free. Additionally, in KS picture the quarks are all in the bi-fundamental representations of the two possible UV gauge groups and they eventually cascade away in the far IR. Also this construction is for zero temperature gauge theories. What we need is a dual gravity theory that allows quarks in the fundamental representation at high temperature. 
The problem of having a fundamental quark can be resolved by inserting $N_{f}$ D7 branes in the KS geometry. This is a subtle issue and so far we only know how to insert coincident D7 branes in the Klebanov-Tseytlin background [39]. The resulting background is the Ouyang background [39] that has all the type IIB fluxes switched on, including the axio-dilaton. Now to switch on a non zero temperature we need to insert a black hole into this background and the Hawking temperature of the black hole will correspond to the gauge theory temperature. Combining all physics ingredients together the metric in OKS-BH geometry looks like

$$
d s^{2}=\frac{1}{\sqrt{h}}\left[-g_{1}(u) d t^{2}+d x^{2}+d y^{2}+d z^{2}\right]+\sqrt{h}\left[g_{2}^{-1}(u) d u^{2}+d \mathcal{M}_{5}^{2}\right]
$$

where $h$ is the warp factor, $g_{i}(u)$ are the black-hole factors, $u$ denotes the extra dimension and $d \mathcal{M}_{5}^{2}$ is the metric of warped resolved-deformed conifold.

The above picture works well at IR. Now we need to see that the theory should become conformal and free at UV. In other words we want AdS-Schwarchild geometry in asymptotic limit. It is clear that we cannot use the pure OKS-BH background and we need to introduce the appropriate UV cap to it. As a consequence, the above metric will receive corrections due to the UV cap as

$$
d s^{2}=\frac{1}{\sqrt{h}}\left[-g(u) d t^{2}+d x^{2}+d y^{2}+d z^{2}\right]+\sqrt{h}\left[g(u)^{-1} g_{u u} d u^{2}+g_{m n} d x^{m} d x^{n}\right]
$$

where we have set black hole factors $g_{1}(u)=g_{2}(u)=g(u)$. The corrections $g_{u u}$ are of the form $u^{-n}$ and appear in the metric because the existence of axio-dilaton and the seven-brane sources tell us that the unwarped metric may not remain Ricci flat. These may be written as

$$
g_{u u}=1+\sum_{i=0}^{\infty} \frac{a_{u u, i}}{u^{i}}
$$

where $a_{u u, i}$ are the coefficients similar to $a_{i}$ and can be solved for exactly as shown in [43].

The warp factor, $h$ can be obtained as

$$
h=\frac{L^{4}}{u^{4}}\left[1+\sum_{i=1}^{\infty} \frac{a_{i}}{u^{i}}\right]
$$

where $a_{i}$ are coefficients of $\mathcal{O}\left(g_{s} N_{f}\right)$ and can be solved for exactly as shown in [43] and $L$ denotes the curvature of space. This metric reduces to OKS-BH in IR and is asymptotically $A d S_{5} \times M_{5}$ in the UV. It describes the geometry all the way from the IR to the UV. 
With the change of coordinates $z=1 / u$, we can rewrite the metric as

$$
d s^{2}=g_{\mu \nu} d X^{\mu} d X^{\nu}=A_{n} z^{n-2}\left[-g(z) d t^{2}+d \vec{x}^{2}\right]+\frac{B_{l} z^{l}}{A_{m} z^{m+2} g(z)} d z^{2}+\frac{1}{A_{n} z^{n}} d s_{M_{5}}^{2},
$$

where $d s_{\mathcal{M}_{5}}^{2}$ is the metric of the internal space and $A_{n}$ 's are the coefficients that can be extracted from the $a_{i}$ 's as follows:

$$
\frac{1}{\sqrt{h}}=\frac{1}{L^{2} z^{2} \sqrt{a_{i} z^{i}}} \equiv A_{n} z^{n-2}=\frac{1}{L^{2} z^{2}}\left[a_{0}-\frac{a_{1} z}{2}+\left(\frac{3 a_{1}^{2}}{8 a_{0}}-\frac{a_{2}}{2}\right) z^{2}+\cdots\right],
$$

which gives $A_{0}=\frac{a_{0}}{L^{2}}, A_{1}=-\frac{a_{1}}{2 L^{2}}, A_{2}=\frac{1}{L^{2}}\left(\frac{3 a_{1}^{2}}{8 a_{0}}-\frac{a_{2}}{2}\right)$ and so on. Note that since $a_{i}$ 's for $i \geq 1$ are of $\mathcal{O}\left(g_{s} N_{f}\right)$ and $L^{2} \propto \sqrt{g_{s} N}$, so in the limit $g_{s} N_{f} \rightarrow 0$ and $N \rightarrow \infty$ all $A_{i}$ 's for $i \geq 1$ are very small. The $u^{-n}$ corrections mentioned above in Eq.(2) are accommodated via $B_{l} z^{l}$ series which is given by

$$
B_{l} z^{l}=1+a_{z z, i} z^{i}
$$

In fact the complete picture can be divided into three regions. Region 1 is the IR region where we have pure OKS-BH geometry. Region 3 is the UV region where UV cap has been added. And region 2 is the interpolating region between UV and IR. The background for all these three regions and the process of adding UV cap has been described in full details in [43]. Also the RG flow associated with these regions and the corresponding field theory realizations have been discussed in [41]. We shall not go into the complete details here and will use the metric given in Eq.(4) in extremizing the action to calculate the potential in the next section.

\section{Heavy quark potential from Gravity}

One of the most important gauge invariant quantities defined in non-Abelian $S U\left(N_{c}\right)$ gauge theories $[45,46]$ is the Wilson loop

$$
W(\mathcal{C})=\frac{1}{N_{c}} \operatorname{tr} P \exp \left[i g \oint_{C} A_{\mu} d x^{\mu}\right]
$$

where $\mathcal{C}$ is a closed loop in a 4-dimensional spacetime, which is usually taken as a rectangular loop of spatial and temporal extensions $r$ and $\mathcal{T}$, respectively. $P$ indicates path-ordering, $g$ is the coupling, $A_{\mu}$ is the non-Abelian gauge field potential operator. The trace (tr) is performed 
over the fundamental representation of the gauge field. The vacuum expectation value ('o') of the Wilson loop gives the desired heavy quark potential $V_{Q \bar{Q}}$ :

$$
\lim _{\mathcal{T} \rightarrow \infty}\langle W(C)\rangle_{0} \sim e^{i \mathcal{T} V_{Q \bar{Q}}(r)}
$$

At finite temperature, the thermal average of the Polyakov loop correlator $\left(\left\langle\operatorname{tr} \mathbf{L}^{\dagger}(r) \operatorname{tr} \mathbf{L}(0)\right\rangle\right)$ gives the free energy of a heavy $Q \bar{Q}$ pair [42] at finite temperature, which is quite often taken as the heavy quark potential at finite temperature on the lattice. However, the potential at finite temperature can also be obtained from the Wilson loop's expectation value evaluated in a thermal state of the gauge theory:

$$
\lim _{\mathcal{T} \rightarrow \infty}\langle W(C)\rangle \sim e^{i \mathcal{T} V_{Q \bar{Q}}(r, T)}
$$

Maldacena was the first to calculate the rectangular Wilson loop in the vacuum of strongly coupled $\mathcal{N}=4$ SYM theory [36] and was later calculated in finite temperature from the correlator of two Polyakov loops in [37, 38]. According to the gauge/gravity prescription [36], the expectation value of $W(C)$ in a strongly coupled gauge theory dual to a theory of gravity is

$$
\langle W(C)\rangle \sim Z_{s t r}
$$

where $Z_{\text {str }}$ is the generating functional of the string in the bulk, having the loop $C$ at the boundary. In the classical gravity approximation

$$
Z_{s t r} \sim e^{i S_{s t r}}
$$

where $S_{\text {str }}$ is the classical string action propagating in the bulk evaluated at an extremum.

In general this heavy quark potential can have an imaginary part [15, 47, 29, 20, 48, 49], which contributes to the thermal decay width and is related to the imaginary part of the gluon self-energy. There are two processes responsible for the thermal width, namely the Landau damping and the singlet to color octet transition. In AdS/CFT framework, people have obtained the non-zero imaginary part for large separation (beyond a critical separation, $r_{c}$ ) by analytically continuing the string configurations into the complex plane [52]. Recently the imaginary contribution has been obtained from the thermal fluctuation around the bottom of the sagging string in the bulk that connects the heavy quarks located at the boundary [50, 51], where the thermal fluctuation causes the maximum length of the sagging classical string to cross the horizon 
of the black hole and makes the potential absorptive. In both cases, the potential is obtained by extremizing the world sheet of open string attached to the heavy quark pair located at the boundary of $\mathrm{AdS}_{5}$ space. Recently a real-time complex potential $V_{Q \bar{Q}}(\mathrm{t}, \mathrm{r})$ is derived from the Wilson loop in the background of AdS black hole by the analytic continuation from the imaginary time to the real time [53], where the complex string configuration has been interpreted as a real string which is moving rather than being static.

Following the background geometry described above in section 2, the Nambu-Goto action can be defined as

$$
S_{N G}=\frac{1}{2 \pi} \int d \sigma d \tau \sqrt{-\operatorname{det}\left[\left(g_{\mu \nu}+\partial_{\mu} \phi \partial_{\nu} \phi\right) \partial_{a} X^{\mu} \partial_{b} X^{\nu}\right]}
$$

where $\phi$ is background dilaton field. It is responsible for breaking of conformal symmetry of theory and is given by

$$
\phi=\log g_{s}-g_{s} D_{n+m_{o}} z^{n+m_{o}}
$$

Using the parametrization $X^{\mu}=(t, x, 0,0, z), \tau=t, \sigma=x$ and $\partial_{a}=\frac{\partial}{\partial \tau}, \partial_{b}=\frac{\partial}{\partial \sigma}$, we extremize the open string worldsheet attached to a static quark at $x=+r / 2$ and an anti-quark at $x=-r / 2$. Using the metric given above in Eq. (4), the Nambu-Goto action can be rewritten as

$$
\begin{aligned}
S_{N G}= & \frac{\mathcal{T}}{2 \pi} \int_{-\frac{r}{2}}^{\frac{r}{2}} \frac{d x}{z^{2}} \\
& \sqrt{\left(A_{n} z^{n}\right)^{2} g(z)+\left(B_{l} z^{l}+2 g(z) g_{s}^{2}\left(n+m_{o}\right) D_{n+m_{o}}\left(l+m_{o}\right) D_{l+m_{o}} A_{k} z^{\left.k+n+l+2 m_{o}\right)\left(z^{\prime}\right)^{2}}\right.}(14
\end{aligned}
$$

where $g(z)=1-\frac{z^{4}}{z_{h}^{4}} \cdot z_{h}=\frac{1}{\pi T}$ is the black hole horizon. By defining

$$
B_{m} z^{m}=B_{l} z^{l}+2 g(z) g_{s}^{2}\left(n+m_{o}\right) D_{n+m_{o}}\left(l+m_{o}\right) D_{l+m_{o}} A_{k} z^{k+n+l+2 m_{o}}
$$

the above action can be written in a closed form

$$
S_{N G}=\frac{\mathcal{T}}{2 \pi} \int_{-\frac{r}{2}}^{\frac{r}{2}} d x \sqrt{\left(A_{n} z^{n}\right)^{2}\left(\frac{1}{z^{4}}-\frac{1}{z_{h}^{4}}\right)+B_{m} z^{m} \frac{\left(z^{\prime}\right)^{2}}{z^{4}}} .
$$

The Nambu-Goto action can also written as an integral over $z$,

$$
S_{N G}=\frac{\mathcal{T}}{\pi} \int_{0}^{z_{\max }} \frac{d z}{z^{\prime}} \sqrt{\left(A_{n} z^{n}\right)^{2}\left(\frac{1}{z^{4}}-\frac{1}{z_{h}^{4}}\right)+B_{m} z^{m} \frac{\left(z^{\prime}\right)^{2}}{z^{4}}}
$$

Since this action does not depend explicitly on $x$, so the corresponding Hamiltonian will be a constant of motion, i.e.,

$$
H=z^{\prime} \frac{\partial L}{\partial z^{\prime}}-L=C_{0}(\text { say })
$$


Thus the constant $C_{0}$ can be determined as

$$
\frac{1}{L}\left(\left(z^{\prime}\right)^{2} \frac{B_{m} z^{m}}{z^{4}}-L^{2}\right)=-\left(\frac{1}{z^{4}}-\frac{1}{z_{h}^{4}}\right) \frac{\left(A_{n} z^{n}\right)^{2}}{L}=C_{0}
$$

Using the fact that at $z=z_{\max }, z^{\prime}=0$, where $z_{\max }$ is the maximum of the string coordinate along fifth dimension, we can find out $z^{\prime}$ by simple algebra

$$
z^{\prime}=\frac{d z}{d x}=\frac{\sqrt{z_{h}^{4}-z^{4}}\left(A_{n} z^{n}\right)}{z_{h}^{2} \sqrt{B_{m} z^{m}}}\left(\frac{\left(z_{h}^{4}-z^{4}\right)\left(A_{n} z^{n}\right)^{2} z_{\max }^{4}}{\left(z_{h}^{4}-z_{\max }^{4}\right)\left(A_{n} z_{\max }^{n}\right)^{2} z^{4}}-1\right)^{\frac{1}{2}}
$$

Integrating both sides, the above equation yields the separation $r$ as

$$
\begin{aligned}
r=\frac{2 z_{h}^{2} \sqrt{z_{h}^{4}-z_{\max }^{4}}\left(A_{n} z_{\max }^{n}\right)}{z_{\max }^{2}} & \int_{0}^{z_{\max }} d z \frac{z^{2} \sqrt{B_{m} z^{m}}}{\left(z_{h}^{4}-z^{4}\right)\left(A_{n} z^{n}\right)^{2}} \times \\
& \left(1-\frac{\left(z_{h}^{4}-z_{\max }^{4}\right)\left(A_{n} z_{\max }^{n}\right)^{2} z^{4}}{\left(z_{h}^{4}-z^{4}\right)\left(A_{n} z^{n}\right)^{2} z_{\max }^{4}}\right)^{-\frac{1}{2}}
\end{aligned}
$$

Expanding the square root and keeping only the first term because the coefficients $A_{i}$ 's are small, the separation $(r)$ becomes

$$
r=\frac{2 z_{h}^{2} \sqrt{z_{h}^{4}-z_{\max }^{4}}\left(A_{n} z_{\max }^{n}\right)}{z_{\max }^{2}} I
$$

where the integral $I$ is defined by

$$
I=\int_{0}^{z_{\max }} d z \frac{z^{2} \sqrt{B_{m} z^{m}}}{\left(z_{h}^{4}-z^{4}\right)\left(A_{n} z^{n}\right)^{2}}
$$

Now we obtain the action by substituting $z^{\prime}$ from Eq.(19) into Eq.(17)

$$
S_{N G}=\frac{\mathcal{T}}{\pi} \int_{0}^{z_{\max }} \frac{d z \sqrt{B_{m} z^{m}}}{z^{2}}\left(1-\frac{\left(z_{h}^{4}-z_{\max }^{4}\right)\left(A_{n} z_{\max }^{n}\right)^{2} z^{4}}{\left(z_{h}^{4}-z^{4}\right)\left(A_{n} z^{n}\right)^{2} z_{\max }^{4}}\right)^{-\frac{1}{2}}
$$

Expanding the square root and keeping only the first two terms (since the coefficients $A_{i}$ 's are small), the action is obtained as

$$
S_{N G}=\frac{\mathcal{T}}{\pi}\left[\int_{0}^{z_{\max }} \frac{d z \sqrt{B_{m} z^{m}}}{z^{2}}+\frac{1}{2} \frac{\left(z_{h}^{4}-z_{\max }^{4}\right)\left(A_{n} z_{\max }^{n}\right)^{2}}{z_{\max }^{4}} I\right]
$$

After substituting the integral $I$ in terms of $r$, the action becomes

$$
\begin{aligned}
S_{N G} & =\frac{\mathcal{T}}{\pi} \int_{0}^{z_{\max }} \frac{d z \sqrt{B_{m} z^{m}}}{z^{2}}+\frac{\mathcal{T}}{4 \pi} \frac{\sqrt{z_{h}^{4}-z_{\text {max }}^{4}}}{z_{h}^{2} z_{\max }^{2}} A_{n} z_{\text {max }}^{n} r \\
& \equiv S^{1}+S^{2}
\end{aligned}
$$


The first term in the action, $S^{1}$ diverges in the lower limit of the integration, so we regularize it by integrating from $\epsilon$, instead of 0 to $z_{\max }[43]$ and identifying the divergent term in the integral

$$
S^{1}=\frac{\mathcal{T}}{\pi} \int_{\epsilon}^{z_{\max }} \frac{d z \sqrt{B_{m} z^{m}}}{z^{2}}
$$

where we take $A_{0}=1$ and $A_{1}=0$ and similarly $B_{0}=1$ and $B_{1}=0$. Let us also assume for simplicity that the higher coefficients $A_{i}$ and $B_{i}$ are very small for $i \geq 3$ and can be neglected. This simplification reduces the series $A_{n} z^{n}$ to $1+A_{2} z^{2}$ and $B_{m} z^{m}$ to $1+B_{2} z^{2}$. This will also simplify all the expressions and help us to keep an analytic control on the equations. In the limit of small coefficients, $S^{1}$ becomes

$$
S^{1}=\frac{\mathcal{T}}{\pi} \int_{\epsilon}^{z_{\max }} \frac{d z\left(1+\frac{B_{2}}{2} z^{2}\right)}{z^{2}}
$$

After integrating and separating into finite and divergent terms, $S^{1}$ becomes in the limit $\epsilon \rightarrow 0$

$$
S^{1}=\lim _{\epsilon \rightarrow 0} \frac{\mathcal{T}}{\pi}\left(-\frac{1}{z_{\max }}+\frac{1}{\epsilon}+\frac{B_{2}}{2}\left(z_{\max }-\epsilon\right)\right)
$$

Therefore subtracting the divergent piece in the limit $\epsilon \rightarrow 0$ from the action, we obtain the renormalised action

$$
S_{N G}^{r e n}=\frac{\mathcal{T}}{4 \pi} \frac{\sqrt{z_{h}^{4}-z_{\max }^{4}}}{z_{h}^{2} z_{\max }^{2}}\left(1+A_{2} z_{\text {max }}^{2}\right) r+\frac{\mathcal{T}}{\pi}\left(-\frac{1}{z_{\max }}+\frac{B_{2}}{2} z_{\max }\right)
$$

and hence the potential is given by

$$
\begin{aligned}
V_{Q \bar{Q}} & =\lim _{\mathcal{T} \rightarrow \infty} \frac{S_{N G}^{r e n}}{\mathcal{T}} \\
& =\frac{1}{4 \pi} \frac{\sqrt{z_{h}^{4}-z_{\max }^{4}}}{z_{h}^{2} z_{\max }^{2}}\left(1+A_{2} z_{\max }^{2}\right) r+\frac{1}{\pi}\left(-\frac{1}{z_{\max }}+\frac{B_{2}}{2} z_{\max }\right)
\end{aligned}
$$

which is a function of both $z_{\max }$ and $\mathrm{r}$. We will therefore express $z_{\max }$ as a function of $\mathrm{r}$ from Eq.(21) and then plug in to the above potential. To do that we first concentrate on the integral $I$, which is simplified into, after neglecting the terms beyond second order:

$$
I=\int_{0}^{z_{\max }} d z \frac{z^{2} \sqrt{1+B_{2} z^{2}}}{\left(z_{h}^{4}-z^{4}\right)\left(1+A_{2} z^{2}\right)^{2}}
$$


In the limit of small coefficients, this integral turns out to be

$$
\begin{aligned}
I & =-\frac{1}{8\left(-1+A_{2}^{2} z_{h}^{4}\right)^{2}}\left[\frac{2\left(2 A_{2}-B_{2}\right)\left(-1+A_{2}^{2} z_{h}^{4}\right) z_{\max }}{\left(1+A_{2} z_{\max }^{2}\right)}\right. \\
& -\frac{2\left(6 A_{2}-B_{2}+A_{2}^{2} z_{h}^{4}\left(2 A_{2}-3 B_{2}\right)\right.}{\sqrt{A_{2}}} \tan ^{-1}\left(\sqrt{A_{2}} z_{\max }\right) \\
& -\frac{2\left(1+A_{2} z_{h}^{2}\right)^{2}\left(-2+B_{2} z_{h}^{2}\right)}{z_{h}} \tan ^{-1} \frac{z_{\max }}{z_{h}} \\
& \left.+\frac{\left.\left(-1+A_{2} z_{h}^{2}\right)^{2}\right)\left(2+B_{2} z_{h}^{2}\right)}{z_{h}} \log \left(\frac{z_{h}-z_{\max }}{z_{h}+z_{\max }}\right)\right]
\end{aligned}
$$

Next we will find the solution for $r$ in two limits, namely $z_{\max }>>z_{h}$ and $z_{\max }<<z_{h}$. For $z_{\max }>>z_{h}$, the separation $r$ from Eq.(21) becomes

$$
r=2 i z_{h}^{2}\left(1-\frac{z_{h}^{4}}{2 z_{\text {max }}^{4}}\right)\left(1+A_{2} z_{\text {max }}^{2}\right) I\left(z_{\max }>>z_{h}\right)
$$

Using the asymptotic expansions of the functions appeared in the integral $I$ :

$$
\begin{gathered}
\tan ^{-1} z(z>>1)=\frac{\pi}{2}-\frac{1}{z}+\frac{1}{3 z^{2}}-\frac{1}{5 z^{5}}+O\left[\frac{1}{z}\right]^{7} \\
\log \left(\frac{\left(\frac{z_{h}}{z_{\max }}-1\right)}{\left(\frac{z_{h}}{z_{\max }}+1\right)}\right)=i \pi-2 \frac{z_{h}}{z_{\max }}-\frac{2}{3} \frac{z_{h}^{3}}{z_{\max }^{3}}-\frac{2}{5} \frac{z_{h}^{5}}{z_{\max }^{5}}-O\left[\frac{z_{h}}{z_{\max }}\right]^{7},
\end{gathered}
$$

the separation $r$ thus becomes as a function of $z_{\max }$

$$
\begin{aligned}
\frac{r}{2 i z_{h}^{2}} & =-\left(c_{1}+i c_{2}\right) A_{2} z_{\max }^{2}-\left(c_{1}+i c_{2}\right)+\frac{B_{2}}{6 A_{2} z_{\max }}+\frac{1}{2} \frac{z_{h}^{4} A_{2}}{z_{\max }^{2}}\left(c_{1}+i c_{2}\right) \\
& +\frac{\left(6 A_{2}-B_{2}\right)}{30 A_{2}^{2} z_{\max }^{3}}+\frac{1}{2} \frac{z_{h}^{4}}{z_{\max }^{4}}\left(c_{1}+i c_{2}\right)
\end{aligned}
$$

where $c_{1}$ and $c_{2}$ are defined as

$$
\begin{aligned}
c_{1} & =\frac{\pi}{8}\left[\frac{1}{z_{h}}\left(\frac{2-B_{2} z_{h}^{2}}{\left(A_{2} z_{h}^{2}-1\right)^{2}}\right)-\frac{6 A_{2}-B_{2}+A_{2}^{2} z_{h}^{4}\left(2 A_{2}-3 B_{2}\right)}{\sqrt{A_{2}}\left(-1+A_{2}^{2} z_{h}^{4}\right)^{2}}\right] \\
c_{2} & =\frac{\pi}{8 z_{h}} \frac{2+B_{2} z_{h}^{2}}{\left(A_{2} z_{h}^{2}+1\right)^{2}}
\end{aligned}
$$

We now invert the series (30) to obtain $z_{\max }$ in terms of $r$ as,

$$
\begin{aligned}
z_{\text {max }} & =\frac{1}{\sqrt{2 A_{2} z_{h}^{2}\left(-i c_{1}+c_{2}\right)}} \sqrt{r}-\sqrt{\frac{\left(-i c_{1}+c_{2}\right) z_{h}^{2}}{2 A_{2}}} \frac{1}{\sqrt{r}}-\frac{i}{6} \frac{B_{2}}{A_{2}} \frac{z_{h}^{2}}{r} \\
& +\frac{\left(\left(-i c_{1}+c_{2}\right) z_{h}^{2}\right)^{3 / 2}\left(-1+2 A_{2}^{2} z_{h}^{4}\right)}{2 \sqrt{2 A_{2}}} \frac{1}{r^{3 / 2}} \\
& +\frac{2\left(3 A_{2}+2 B_{2}\right)\left(-c_{1}-i c_{2}\right)}{15 A_{2}} \frac{z_{h}^{4}}{r^{2}}+O\left[\frac{1}{r}\right]^{5 / 2}
\end{aligned}
$$


which shows that the string coordinates become complex and hence the potential becomes imaginary. We have checked numerically that this situation corresponds to $r>r_{c}$, where $r_{c}$ is some critical separation and for this geometry it turns out to be $0.53 z_{h}$. However, the authors of $[38,37]$ abandoned the solution for $r>r_{c}$ but the authors in [52] suggested that the complexvalued saddle points beyond $r_{c}\left(=0.87 z_{h}\right)$ may be interpreted as the quasi-classical configurations in the classically forbidden region of string coordinates, analogous to the method of complex trajectories used in quasi-classical approximations to quantum mechanics [54]. The complexification of the string coordinates simply indicates that the saddle point of the integral over string coordinates becomes complex. According to the standard AdS/CFT prescription [36], in the large- $N_{c}$ large- $\lambda$ limit the integral over string coordinates is still dominated by the saddle point, even if it is complex so the saddle point approximations is still valid [52]. Recently authors in [53] estimated the critical length scale $0.62 z_{h}$, in the same sense of [52], with an interpretation of the complex string configuration as a real string which is moving rather than being static.

In this limit $\left(z_{\max }>>z_{h}\right)$, the potential reduces to

$$
V_{Q \bar{Q}}=\frac{1}{\pi}\left(\frac{B_{2}}{2} z_{\max }-\frac{1}{z_{\max }}\right)-\frac{i r}{4 \pi z_{h}^{2}}\left(A_{2} z_{\text {max }}^{2}+1-\frac{A_{2}}{2} \frac{z_{h}^{4}}{z_{\max }^{2}}-\frac{1}{2} \frac{z_{h}^{4}}{z_{\max }^{4}}\right)
$$

Here we could have chosen either $+i$ or $-i$ in the second term above. To select the correct sign we note that time evolution operator in quantum mechanics is $e^{-i E t} \sim e^{\operatorname{Im}[E] t}$. To maintain unitarity we demand that the $\operatorname{Im}[\mathrm{V}(\mathrm{r})]$ should be negative. When we demand this we find that we should use a negative sign here.

Finally we substitute for $z_{\max }$ in terms of $\mathrm{r}$ from Eq.(32), to obtain the potential as a function of $r$ and $T$ only:

$$
\begin{aligned}
V_{Q \bar{Q}}(r, T)= & -\frac{i}{8 \pi\left(-i c_{1}+c_{2}\right)} \frac{r^{2}}{z_{h}^{4}}+\frac{5}{12 \sqrt{2}} \frac{B_{2}}{\sqrt{A_{2}\left(-i c_{1}+c_{2}\right)}} \frac{\sqrt{r}}{\pi z_{h}} \\
& -\frac{9}{20 \sqrt{2}} \frac{\left(4 A_{2}+B_{2}\right) \sqrt{-i c_{1}+c_{2}}}{\sqrt{A_{2}}} \frac{z_{h}}{\pi \sqrt{r}}-\frac{13 i}{72} \frac{B_{2}^{2}}{A_{2} \pi} \frac{z_{h}^{2}}{r}
\end{aligned}
$$

We can split it into real and imaginary parts, where the real part is given by (a dimensionless variable, $\hat{r}=r T$ )

$$
\begin{aligned}
& \operatorname{Re}\left[V_{Q \bar{Q}}\right](\hat{r}, T) \stackrel{z_{\max } \gg z_{h}}{\simeq} \frac{1}{8} \frac{c_{1}}{c_{1}^{2}+c_{2}^{2}} \pi^{3} T^{2} \hat{r}^{2}+\frac{5}{12 \sqrt{2} \frac{B_{2}}{\sqrt{A_{2}}} \frac{1}{\sqrt{c_{1}^{2}+c_{2}^{2}}} \sqrt{\frac{\sqrt{c_{1}^{2}+c_{2}^{2}}+c_{2}}{2}} \sqrt{T \hat{r}}} \\
&-\frac{9}{20 \sqrt{2}} \frac{\left(4 A_{2}+B_{2}\right)}{\sqrt{A_{2}}} \sqrt{\frac{\sqrt{c_{1}^{2}+c_{2}^{2}}+c_{2}}{2} \frac{1}{\pi^{2} \sqrt{T \hat{r}}}}
\end{aligned}
$$


For small $r$, the first two terms (positive) tend to zero while the third term becomes (negative) large, hence the potential is highly attractive. For large $r$, the first two terms (positive) become larger while the third term (negative) becomes smaller, hence the potential goes to continuum. As the temperature increases the first two terms becomes larger, hence the potential becomes quickly zero for smaller $\mathrm{r}$. This can be understood by the fact that as the temperature increases, screening becomes stronger.

The imaginary part upto leading order in $r$ is given by

$$
\begin{aligned}
\operatorname{Im}\left[V_{Q \bar{Q}}\right](\hat{r}, T) & =-\frac{1}{8} \frac{c_{2}}{c_{1}^{2}+c_{2}^{2}} \pi^{3} T^{2} \hat{r}^{2} \\
& +\frac{5}{12 \sqrt{2}} \frac{B_{2}}{\sqrt{A_{2}}} \frac{1}{\sqrt{c_{1}^{2}+c_{2}^{2}}} \sqrt{\frac{\sqrt{c_{1}^{2}+c_{2}^{2}}-c_{2}}{2}} \sqrt{T \hat{r}}
\end{aligned}
$$

which shows that the imaginary part vanishes for small $r$ and agrees with the perturbative result.

We will now find the potential for the other extreme limit i.e.., very small $z_{\max }$ and $z_{\max }<<$ $z_{h}$. In this limit, the separation $r$ becomes

$$
r=\left(\frac{2 z_{h}^{4}}{z_{\max }^{2}}-z_{\max }^{2}\right)\left(1+A_{2} z_{\max }^{2}\right) I\left(z_{\max }<<z_{h}\right)
$$

Using the expansion of $\tan ^{-1}$ and $\log$ functions in the integral $I\left(z_{\max }<<z_{h}\right)$

$$
\begin{gathered}
\tan ^{-1} z(z<<1)=z-\frac{1}{3} z^{3}+\frac{1}{5} z^{5}+O[z]^{7} \\
\log \left(\frac{1-\frac{z_{\text {max }}}{z_{h}}}{1+\frac{z_{\max }}{z_{h}}}\right)=-2 \frac{z_{\max }}{z_{h}}-\frac{2}{3} \frac{z_{\text {max }}^{3}}{z_{h}^{3}}-\frac{2}{5} \frac{z_{\text {max }}^{5}}{z_{h}^{5}}+O\left[\frac{z_{\text {max }}}{z_{h}}\right]^{7}
\end{gathered}
$$

the separation $r$ can be expressed in a series of $z_{\max }$ from Eq. (37)

$$
r=\frac{2}{3} z_{\max }+\frac{3 B_{2}-2 A_{2}}{15} z_{\text {max }}^{3}+\frac{-5+6 A_{2}^{2} z_{h}^{4}-9 A_{2} B_{2} z_{h}^{4}}{105 z_{h}^{4}} z_{\text {max }}^{5},
$$

which will eventually be inverted to express $z_{\max }$ in terms of $r$ as

$$
z_{\max }=\frac{3 r}{2}+\frac{27}{80}\left(2 A_{2}-3 B_{2}\right) r^{3}+O[r]^{5}
$$

In this limit, the potential in Eq.(25) reduces to

$$
V_{Q \bar{Q}}=\frac{1}{\pi}\left(\frac{B_{2}}{2} z_{\max }-\frac{1}{z_{\max }}\right)+\frac{r}{4 \pi}\left(\frac{1}{z_{\max }^{2}}+A_{2}-\frac{1}{2} \frac{z_{\max }^{2}}{z_{h}^{4}}-\frac{A_{2}}{2} \frac{z_{\max }^{4}}{z_{h}^{4}}\right)
$$


After plugging $z_{\max }$ from (39), the potential in $z_{\max }<<z_{h}$ limit becomes

$$
\operatorname{Re}\left[V_{Q \bar{Q}}\right](r, T) \stackrel{z_{\max } \ll z_{h}}{\simeq}-\frac{5}{9 \pi r}+\frac{9\left(A_{2}+B_{2}\right)}{20 \pi} r+\frac{27}{224 \pi}\left(\frac{\left(2 A_{2}-B_{2}\right)\left(2 A_{2}+7 B_{2}\right)}{50}-\frac{1}{z_{h}^{4}}\right) r^{3}+O[r]^{5}
$$

The above form of potential looks like a (Cornell) potential at $T=0$, apart from a tiny $r^{3}$ temperature-dependent term, which can be understood by the fact that in small distance limit $(r T<<1), Q \bar{Q}$ pair does not see the medium. Note that there is no imaginary part in this limit, as expected. Since we have the potential in both asymptotic limits, we can interpolate them to find a general expression for the potential

$$
\operatorname{Re}\left[V_{Q \bar{Q}}\right](r, T) \approx \frac{5}{9 \pi r} \frac{\left(r-r_{0}\right)^{3}}{r_{0}^{3}}
$$

where $r_{0}$ is the new scale and is given by

$$
r_{0}=\left(\frac{40\left(c_{1}^{2}+c_{2}^{2}\right)}{9 c_{1} \pi^{4} T^{4}}\right)^{1 / 3}
$$

This formula reduces to $-1 / r$ form for $r<<r_{0}$ and reduces to $r^{2}$ form for $r>>r_{0}$. The parameter $r_{0}$ can be interpreted as the screening length and it's dependence on the temperature can be seen from Eq. (31) and (43). We have evaluated both real (42) and imaginary parts (36) of the potential for increasing temperatures (Fig.1), to see how the potential obtained via gauge-gravity duality in the strong coupling regime gets screened with the increase in temperature.

\section{Conclusion and Discussions}

We have obtained a heavy quark potential at finite temperature in a dual gravity which is somewhat closer to QCD than pure AdS geometry in the sense that it accounts for RG flow. For the small separation limit $(r T<<1$ ) (which implies either $r \rightarrow 0$ or $T \rightarrow 0$ ), the potential looks like a Cornell potential at $T=0$, with a small higher-order confining term, whereas at large separation $(r T>>1)$, the potential gets screened, having a quadratic term, a $\sqrt{r}$ term and an attractive $1 / \sqrt{r}$ term. The quadratic term is probably originated from the non-dominant cubic term at small separation. In addition to it, the potential develops an imaginary term above some separation, $r_{c}=0.53 z_{h}$. The difference in the estimate of critical separations in other calculations $[52,53]$ may be simply due to the different world sheet configuration taken. 

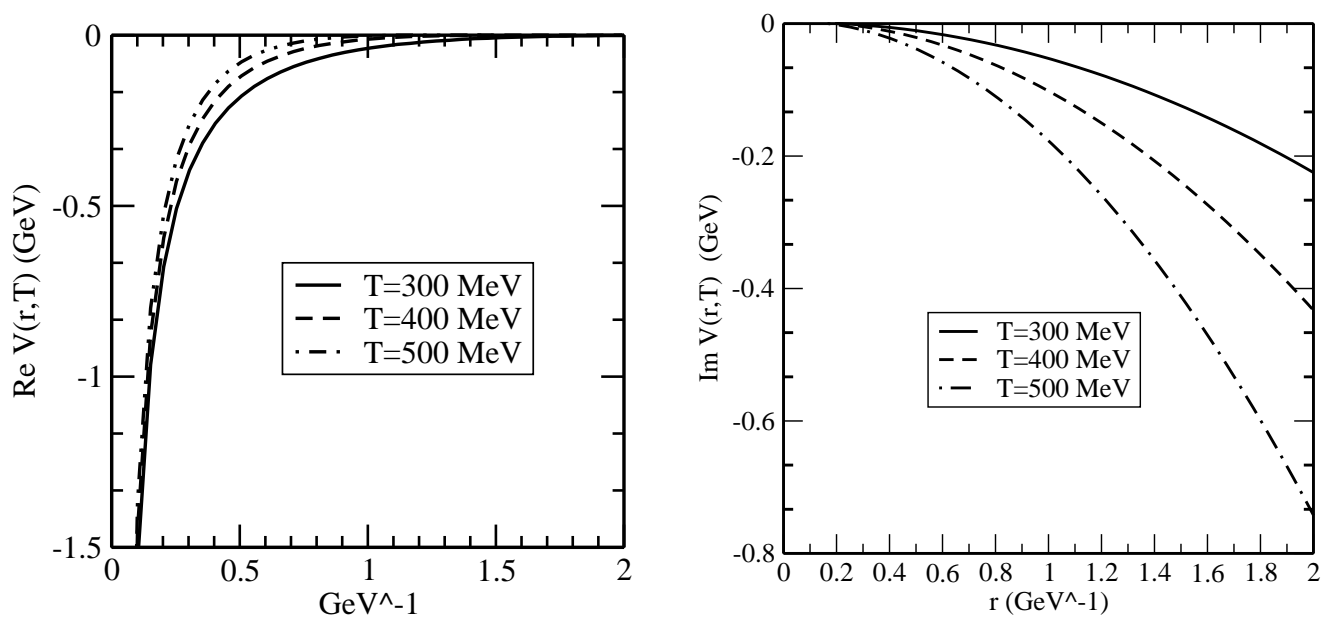

Figure 1: Variations of the real and imaginary part of the potential with the separation $r$, where the value of both coefficients $A_{2}$ and $B_{2}$ are taken as 0.24 .

Moreover we found that the screening of potential is in the form of a power law (which means $\operatorname{Re}[V(r)]$ falls off as some power of $1 / r T)$ in our case, unlike the exponential falloff due to debye screening in the long distance in which $\operatorname{Re}[V(r)] \sim \exp \left(-m_{D} r\right)$.

\section{Acknowledgements}

We would like to thank Yuri V. Kovchegov for his extremely helpful comments and suggestions and also for patiently explaining his paper. We would also like to thank Keshav Dasgupta for looking through the calculations. B. K. P is thankful to the CSIR project (No. 03/1215), Government of India, for the financial assistance..

\section{References}

[1] T. Matsui and H. Satz, Phys. Lett. B 178, 416 (1986).

[2] F. Karsch, M. Mehr, and H. Satz, Z. Phys. C C37, 617 (1988).

M. Asakawa et al. Nucl.Phys. A 715,863 (2003).

[3] M. Asakawa and T. Hatsuda, Phys. Rev. Lett. 92, 012001 (2004).

[4] S. Datta, F. Karsch, P. Petreczky, and I. Wetzorke, Phys. Rev. D 69, 094507 (2004). 
[5] T. Umeda, K. Nomura, and H. Matsufuru, Eur. Phys. J. C 39, 9 (2005).

[6] H. Iida, T. Doi, N. Ishii, H. Suganuma, and K. Tsumura, Phys. Rev. D 74, 074502 (2006).

[7] A. Jakovac, P. Petreczky, K. Petrov, and A. Velytsky, Phys. Rev. D 75, 014506 (2007).

[8] G. Aarts, C. Allton, M. B. Oktay, M. Peardon, and J.-I. Skullerud, Phys. Rev. D 76, 094513 (2007).

[9] S. Digal, P. Petreczky, and H. Satz, Phys. Lett. B 514, 57 (2001); Phys. Rev. D 64, 094015 (2001).

[10] E. V. Shuryak and I. Zahed, Phys. Rev. D 70, 054507 (2004).

[11] D. Cabrera and R. Rapp, Phys.Rev. D 76 ,114506 (2007).

[12] W. M. Alberico, A. Beraudo, A. De Pace, and A. Molinari, Phys. Rev. D 75, 074009 (2007).

[13] A. Mocsy and P. Petreczky, Phys. Rev. D 77, 014501 (2008).

[14] Yu. A. Simonov, Phys. Lett. B 619, 293 (2005).

[15] M. Laine, O. Philipsen, P. Romatschke, and M. Tassler, JHEP 03, 054 (2007).

[16] M. Laine, JHEP 0705, 028 (2007).

[17] A. Mocsy and P. Petreczky, Phys. Rev. D 73, 074007 (2006).

[18] C. Y. Wong and H. W. Crater, Phys. Rev. D 75, 034505 (2007).

[19] N. Brambilla, J. Ghiglieri, A. Vairo, and P. Petreczky, Phys. Rev. D 78, 014017, 2008.

[20] N. Brambilla, M. A. Escobedo, J. Ghiglieri, J. Soto, and A. Vairo, JHEP 1009, 038 (2010).

[21] N. Brambilla, A. Pineda, J. Soto, and A. Vairo, Rev. Mod. Phys. 77, 1423 (2005).

[22] A. Pineda, Prog. Part. Nucl. Phys. 67, 735 (2012).

[23] M. Laine, O. Philipsen, P. Romatschke, and M. Tassler, JHEP 0703, 054 (2007).

[24] M. Laine, Nucl. Phys. A 820, 25C (2009). 
[25] N. Brambilla, M. A. Escobedo, J. Ghiglieri, and A. Vairo, JHEP 1305, 130 (2013).

[26] V. Agotiya, V. Chandra and B. K. Patra, Phys. Rev. C 80, 025210 (2009).

[27] Lata Thakur, N. Haque, Uttam Kakade and B. K. Patra, Phys.Rev. D 88 , 054022 (2013).

[28] Lata Thakur, Uttam Kakade and B. K. Patra, Phys. Rev. D 89 , 094020 (2014).

[29] P. Petreczky, C. Miao, and A. Mocsy, Nucl.Phys., A 855, 125 (2011).

[30] M. Mannarelli and R. Rapp, Phys. Rev. C 72, 064905 (2005).

[31] Y. Burnier and A. Rothkopf, Phys. Rev. D 86, 051503 (2012).

[32] J. M. Maldacena, Adv. Theor. Math. Phys. 2, 231 (1998) [Int. J. Theor. Phys. 38, 1113 (1999)];

[33] E. Witten, Adv. Theor. Math. Phys. 2, 253 (1998); 2, 505 (1998).

[34] S. S. Gubser, I. R. Klebanov and A. M. Polyakov, Phys. Lett. B 428, 105 (1998).

[35] D Teaney, Phys Rev D 68, 034913 (2003).

[36] J. M. Maldacena, Phys. Rev. Lett. 80, 4859 (1998).

[37] A. Brandhuber, N. Itzhaki, J. Sonnenschein and S. Yankielowicz, Phys. Lett. B 434, 36 (1998).

[38] S. -J. Rey, S. Theisen and J. -T. Yee, Nucl. Phys. B 527, 171 (1998).

[39] I. R. Klebanov and M. J. Strassler, JHEP 0008, 052 (2000).

C. Vafa, J. Math. Phys. 42, 2798 (2001).

J. M. Maldacena and C. Nunez, Phys. Rev. Lett. 86, 588 (2001).

P. Ouyang, Nucl. Phys. B 699, 207 (2004).

I. R. Klebanov and A. A. Tseytlin, Nucl. Phys. B 578, 123 (2000).

[40] M. Mia, K. Dasgupta, C. Gale and S. Jeon, Nucl.Phys. B 839, 187 (2010).

[41] F. Chen, L. Chen, K. Dasgupta, M. Mia, and O. Trottier, Phys. Rev. D 87, 041901 (2013). 
[42] L. D. McLerran and B. Svetitsky, Phys. Rev. D 24, 450 (1981).

[43] M. Mia, K. Dasgupta, C. Gale and S. Jeon, Phys. Rev. D 82, 026004 (2010).

[44] M. Mia, K. Dasgupta, C. Gale and S. Jeon, Phys Lett. B 694, 460 (2011).

[45] K. G. Wilson, Phys. Rev. D 10, 2445 (1974).

[46] J. -L. Gervais and A. Neveu, Nucl. Phys. B 163, 189 (1980); A. M. Polyakov, Nucl. Phys. B 164, $171(1980)$.

[47] A. Beraudo, J. P. Blaizot and C. Ratti, Nucl. Phys. A 806, 312 (2008)

[48] Y. Burnier, M. Laine, and M. Vepsalainen, Phys Lett. B678 (2009) 86; A. Dumitru, Y. Guo, and M. Strickland, Phys. Rev. D 79, 114003 (2009).

[49] A. Rothkopf, T. Hatsuda, S. Sasaki, Phys. Rev. Lett. 108, 162001 (2012)

[50] J. Noronha and A. Dumitru, Phys. Rev. Lett. 103, 152304 (2009).

[51] Stefano I. Finazzo, Jorge Noronha, JHEP 1311, 042 (2013).

[52] Javier L. Albacete, Yuri V. Kovchegov, A Taliotis, Phys.Rev. D 78, 115007 (2008).

[53] T Hayata, K Nawa, T Hatsuda, Phys.Rev. D 87, 101901 (2013).

[54] L. D.Landau and E. M. Lifshitz, Quantum mechanics, non-relativistic theory, (ButterworthHeinemann, 2003), vol. 3, ch. 131. 